\title{
Efecto de la Viscosidad y de la Densidad de Mezcla en el Gradiente de Presión de Flujo Homogéneo Gas-Líquido en Tuberías Horizontales
}

\author{
Janneth M. García ${ }^{1}$ y Francisco García ${ }^{2}$ \\ (1) Universidad Simón Bolívar, Departamento de Termodinámica y Fenómenos de Transferencia, \\ Apartado Postal 89000 - Cable Unibolivar-Baruta 1080, Caracas-Venezuela \\ (e-mail: jmgferrer@usb.ve) \\ (2) Universidad Central de Venezuela, Escuela de Ingeniería Mecánica, Apartado Postal 48222, \\ Los Chaguáramos 1041-A, Caracas-Venezuela (e-mail: francisco.garcia@ucv.ve)
}

\begin{abstract}
Resumen
En este trabajo se evalúa la precisión de 76 modelos homogéneos de gradiente de presión para determinar el gradiente de presión experimental reportado en una base de 2415 datos de flujos bifásico gas-líquido en tuberías horizontales. Estos modelos se formularon a partir de 4 ecuaciones de densidad de mezcla combinadas con 19 ecuaciones de viscosidad de mezcla. Al estudiar el efecto de la viscosidad y de la densidad de mezcla en el gradiente de presión, utilizando el modelo homogéneo, se observa que el mejor modelo obtuvo un error absoluto promedio de $25.7 \%$, mientras que el peor modelo obtuvo un error cercano al 2000\%. Los resultados obtenidos indican que la precisión del modelo homogéneo de gradiente de presión de flujo bifásico gas-líquido, depende significativamente de la adecuada selección de las ecuaciones de viscosidad y de densidad de mezcla.
\end{abstract}

Palabras clave: gradiente de presión, viscosidad de mezcla, densidad de mezcla, flujo gas-líquido

\section{Effect of the Mixture Viscosity and the Mixture Density on the Pressure Gradient of Gas-Liquid Homogeneous Flow in Horizontal Pipelines}

\begin{abstract}
In this work the performance of 76 pressure gradient homogeneous models is evaluated in order to determine the experimental pressure gradient reported in a database consisting of 2415 gas-liquid two-phase flow experiments in horizontal pipes. These models were formulated starting from 4 mixture density equations combined with 19 mixture viscosity equations. To study the mixture viscosity and the mixture density effect in the pressure gradient, using the homogeneous model, it is observed that the best model gave an average absolute error of $25.7 \%$, while the worst model obtained an error close to $2000 \%$. The obtained results indicate that the performance of the gasliquid two-phase flow pressure gradient homogeneous model depends significantly on the appropriate selection of the mixture viscosity and the mixture density equations.
\end{abstract}

Keywords: pressure gradient, mixture viscosity, mixture density, gas-liquid flow 


\section{INTRODUCCIÓN}

El flujo bifásico de gas y de líquido en tuberías, se presenta con frecuencia en una gran diversidad de procesos asociados a la industria petrolera, química y nuclear, entre otras. La exactitud en la determinación del gradiente de presión en flujo bifásico de gas y de líquido, es un requisito importante para el diseño y la operación de sistemas donde este tipo de flujo esté presente. Debido a la complejidad de este tipo de flujo, en general, los modelos de predicción de gradiente de presión comúnmente utilizados presentan diferencias entre ellos que pueden superar el 50\% (García et al., 2007; Moreno y Thome, 2007a, 2007b; Spedding et al., 2006).

La dificultad del estudio del flujo bifásico de gas y de líquido en tuberías radica principalmente en las distintas configuraciones espaciales que adquieren los fluidos dentro de la misma y a que la fase gaseosa es compresible. Estas configuraciones espaciales se denominan patrones de flujos. Sin embargo, se han desarrollado modelos de flujo bifásico que son independientes del patrón de flujo y tratan a la mezcla como un fluido como seudo homogéneo. Entre estos modelos se encuentra el modelo de Wallis (1969), en éste las propiedades físicas y la velocidad son consideradas como valores promedios entre ambas fases.

La incidencia de las propiedades de mezcla, en la evaluación del gradiente de presión de flujo bifásico de gas y de líquido en tuberías, a través de un modelo homogéneo, no está claramente establecida. Resultados reportados por García et al. $(2003,2007)$ reportan diferencias significativas en la predicción del gradiente de presión entre modelos homogéneos cuya única diferencia es la forma de evaluar la viscosidad de mezcla. Por ejemplo, los modelos de McAdams et al. (1942) y de Cicchitti et al. (1960), presentan errores porcentuales absolutos promedios de $38 \%$ y $275 \%$, respectivamente, al comparar los valores de gradiente de presión calculados con los experimentales. Haoulo et al. (2005), evaluaron el efecto de las propiedades de mezcla en el gradiente de presión total de un flujo seudo homogéneo de gas y de líquido; sin embargo, los resultados obtenidos en este trabajo no fueron concluyentes debido a que la base de datos experimentales utilizadas tenía un intervalo de aplicación muy limitado y en su mayoría aire-agua. Por otra parte, los resultados reportador por, Saisorn y Wongwises (2008), evidencia un efecto significativo de la viscosidad de la mezcla, en la predicción del gradiente de presión mediante un modelo homogéneo, de flujo bifásico en micro canales circulares horizontales.

En este trabajo, se evalúa el efecto en la determinación del gradiente de presión de 76 modelos, comparando los valores calculados con la caída de presión experimental de una base de 2415 datos de flujo bifásico de gas y de líquido con un amplio intervalo de operación, que incluye diferentes tipos de líquidos con viscosidades que van desde 0.7 a $1118.2 \mathrm{mPa}$.s. Estos modelos se construyeron a partir de 4 ecuaciones de densidad de mezcla combinadas con 19 ecuaciones de viscosidad de mezcla. Adicionalmente, se estudia el comportamiento de las ecuaciones de densidades y de viscosidades de la mezcla, respecto a los valores de las propiedades del líquido y el gas.

\section{MODELO MATEMÁTICO}

En el modelo homogéneo empleado por Wallis (1969) para flujo unidimensional, permanente e isotérmico en tuberías, el gradiente de presión total es evaluado como:

$$
-\frac{d p}{d z}=\frac{P \tau_{W}}{A}+\rho_{M} U_{M} \frac{d U_{M}}{d z}+\rho_{M} g \operatorname{sen} \theta
$$

donde $U_{M}$ y $\rho_{M}$ son la velocidad y la densidad de la mezcla, respectivamente; $A, P$ y $\theta$ son el área de la sección transversal, el perímetro y el ángulo de inclinación de la tubería, respectivamente; $d P / d z$ es el gradiente de presión en la dirección del flujo, $\tau_{w}$ es el esfuerzo de corte en la pared de la tubería y $g$ es la aceleración de gravedad. En el caso de tuberías horizontales el gradiente de presión debido a los cambios de energía potencial, correspondiente al tercer término del lado derecho de la ecuación, es cero. El gradiente de presión por fricción debido al esfuerzo de corte de la seudo fase con la pared interna del tubo, puede ser expresado como: 


$$
\left.\frac{d p}{d z}\right|_{F}=\frac{2}{D} f_{f} \rho_{M} U_{M}^{2}
$$

donde $f_{f}$ es el factor de fricción de Fanning determinado en base al número de Reynolds de mezcla $\operatorname{Re}_{M}=\rho_{M} U_{M} D / \mu_{M}$, siendo $\mu_{M}$ la viscosidad dinámica de la mezcla.

En tuberías horizontales, el gradiente de presión longitudinal debido a los cambios de energía cinética, correspondiente al segundo término del lado derecho de la ecuación (1), generalmente se considera despreciable. Este término puede ser expresado en función de la tasa de flujo másico de mezcla $\dot{m}_{M}=A \rho_{M} U_{M}$,

$$
\left.\frac{d p}{d z}\right|_{A}=\left(\frac{\dot{m}_{M}}{A}\right)^{2} \frac{d}{d z}\left(\frac{1}{\rho_{M}}\right)
$$

La ec. (3) varía en función de la ecuación de densidad de mezcla utilizada. Para este trabajo se utilizaron cuatro ecuaciones de densidad de mezcla, las cuales se muestran en la Tabla 1.

Tabla 1: Ecuaciones de densidad de mezcla

\begin{tabular}{llc}
\hline Autor o fuente & Ecuación de Densidad de Mezcla & Código \\
\hline Dukler et al. (1964) & $\rho_{M}=\frac{\rho_{L} \lambda_{L}^{2}}{H_{L}}+\rho_{G} \frac{\left(1-\lambda_{L}\right)^{2}}{\left(1-H_{L}\right)}$ & $\mathrm{d} 3$ \\
Oliemans (1976) & $\rho_{M}=\frac{\rho_{L} \lambda_{L}+\rho_{G}\left(1-H_{L}\right)}{1-H_{L}+\lambda_{L}}$ & $\mathrm{~d} 4$ \\
Beattie y Whalley (1982) & $\frac{1}{\rho_{M}}=\frac{x}{\rho_{G}}+\frac{1-x}{\rho_{L}}$ & $\mathrm{~d} 1$ \\
Ouyang (1998) & $\rho_{M}=\rho_{L} H_{L}+\rho_{G}\left(1-H_{L}\right)$ & $\mathrm{d} 2$ \\
\hline
\end{tabular}

Siendo $\rho_{L}$ y $\rho_{G}$ las densidades del líquido y del gas, respectivamente; $\lambda_{L}$ y $H_{L}$ son las fracciones volumétricas de líquido sin y con deslizamiento, respectivamente y $x$ es la calidad.

En las ecuaciones de propiedades de mezcla que se requiere conocer la fracción volumétrica de líquido con deslizamiento $H_{L}$, ésta es evaluada a partir de la correlación de Eaton et al. (1967). El código presentado en la tercera columna, se utiliza para identificar los modelos de acuerdo a la ecuación de densidad de mezcla. El cálculo de la viscosidad de la mezcla se realizó con 19 ecuaciones presentes en la literatura especializada, las cuales se listan en la Tabla 2, donde $\mu_{L}$ y $\mu_{G}$ corresponden a las viscosidades del líquido y del gas, respectivamente y $\dot{m}_{M}, \dot{m}_{G}$ y $\dot{m}_{L}$ son el flujo másico de la mezcla, del gas y del líquido, respectivamente.

El proceso de identificación de cualquiera de las combinaciones está realizado como se indican en el siguiente ejemplo; la combinación de la correlación de la viscosidad de mezcla de Dukler et al. (1964), con la combinación de la ecuación de la densidad de mezcla de Oliemans (1976), se representa como Dukler-d4, indicando primero la ecuación de viscosidad utilizada y luego la de densidad de mezcla. De la combinación de las diferentes ecuaciones se generaron 76 modelos, donde se combinan las 4 ecuaciones de densidad de mezcla con las 19 ecuaciones de viscosidad de mezcla presentadas en este trabajo.

\section{RESUMEN BASE DE DATOS}

Los datos experimentales de flujo bifásico de gas y de líquido utilizados para la evaluación, son los reportados por García et al. (2007). De los 2560 datos utilizados originalmente por García et al. 
(2007), no se consideraron los 51 datos de Eaton (1966) y los 94 datos de Pereyra et al. (2001) debido a que la fase gaseosa era gas natural. En la Tabla 3, se presenta un resumen de los 2415 datos experimentales utilizados para evaluar la precisión de los diferentes modelos en la determinación del gradiente de presión de flujo de gas y de líquido en tuberías horizontales.

Tabla 2: Ecuaciones de viscosidad de mezcla

\begin{tabular}{|c|c|}
\hline Autor o fuente & Ecuación de Viscosidad de Mezcla \\
\hline Arrhenius (1887) & $\mu_{M}=\mu_{L}^{\lambda_{L}} \mu_{G}^{\left(1-\lambda_{L}\right)}$ \\
\hline Bingham (1906) & $\mu_{M}=\left[\frac{\lambda_{L}}{\mu_{L}}+\frac{\left(1-\lambda_{L}\right)}{\mu_{G}}\right]^{-1}$ \\
\hline Einstein (1909) & $\mu_{M}=\mu_{L}\left(1+k \lambda_{L}\right)$ \\
\hline Hatschek (1928) & $\mu_{M}=\frac{\mu_{L}}{1-\sqrt[3]{\lambda_{L}}}$ \\
\hline Taylor (1932) & $\mu_{M}=\mu_{L}\left(1+2.5\left(\frac{\mu_{G}+0.4 \mu_{L}}{\mu_{G}+\mu_{L}}\right) \lambda_{L}\right)$ \\
\hline Richardson (1933) & $\mu_{M}=\mu_{L} \exp \left(k \lambda_{L}\right)$ \\
\hline McAdams et al. (1942) & $\frac{1}{\mu_{M}}=\frac{1}{\mu_{G}} \frac{\dot{m}_{G}}{\dot{m}_{M}}+\frac{1}{\mu_{L}}\left(1-\frac{\dot{m}_{G}}{\dot{m}_{M}}\right)$ \\
\hline Vermeulen et al. (1955) & $\mu_{M}=\frac{\mu_{L}}{1-\lambda_{L}}\left[1+\left(\frac{1.5 \mu_{G} \lambda_{L}}{\mu_{L}+\mu_{G}}\right)\right]$ \\
\hline Hoogendoorn (1959) & $\mu_{M}=\mu_{L}^{H_{L}} \mu_{G}^{\left(1-H_{L}\right)}$ \\
\hline Cicchitti et al. (1960) & $\mu_{M}=\mu_{G} \frac{\dot{m}_{G}}{\dot{m}_{M}}+\mu_{L}\left(1-\frac{\dot{m}_{G}}{\dot{m}_{M}}\right)$ \\
\hline Bankoff (1960) & $\mu_{M}=H_{L} \mu_{L}+\left(1-H_{L}\right) \mu_{G}$ \\
\hline Dukler et al. (1964) & $\mu_{M}=\mu_{A} \lambda_{L}+\mu_{B}\left(1-\lambda_{L}\right)$ \\
\hline Cengel (1967) & $\mu_{M}=\mu_{L}\left(1+2.5 \lambda_{L}-11.01 \lambda_{L}^{2}+52.62 \lambda_{L}^{3}\right)$ \\
\hline Soot (1971) & $\mu_{M}=\mu_{L}\left[1+\lambda_{L}\left(\frac{\rho_{G}}{\rho_{L}}-1\right)\right]$ \\
\hline Oliemans (1976) & $\mu_{M}=\frac{\mu_{L} \lambda_{L}+\mu_{G}\left(1-H_{L}\right)}{1-H_{L}+\lambda_{L}}$ \\
\hline Oglesby (1979) & $\mu_{M}=\mu_{L} \exp \left[\left(\frac{\mu_{G}+0.4 \mu_{L}}{\mu_{G}+\mu_{L}}\right)\left(\lambda_{L}+\lambda_{L}^{1.667}+\lambda_{L}^{3.66}\right)\right]$ \\
\hline Beattie y Whalley (1982) & $\mu_{\mathrm{M}}=\mu_{L}(1-\beta)(1+2.5 \beta)+\mu_{G} \beta, \beta=\frac{\rho_{L} x}{\rho_{L} x+\rho_{G}(1-x)}$ \\
\hline Lin et al. (1991) & $\mu_{M}=\frac{\mu_{L} \mu_{G}}{\mu_{G}+x^{1.4}\left(\mu_{L}-\mu_{G}\right)}$ \\
\hline Fourar y Bories (1995) & $\mu_{M}=\lambda_{L} \mu_{L}+\left(1-\lambda_{L}\right) \mu_{G}+2 \sqrt{\lambda_{L} \cdot\left(1-\lambda_{L}\right) \mu_{L} \mu_{G}}$ \\
\hline
\end{tabular}

De acuerdo al conocimiento de los autores, ésta es la base de datos de flujo bifásico de gas y de líquido en tuberías horizontales, que tiene el mayor intervalo de condiciones de operación y propiedades de fluidos compilada y utilizada para evaluar el efecto de las propiedades de mezcla en la determinación del gradiente de presión considerando un modelo de flujo homogéneo. 
Tabla 3: Resumen de los 2415 datos experimentales procesados

\begin{tabular}{llllll}
\hline Variable & Promedio & $\begin{array}{l}\text { Desviación } \\
\text { estándar }\end{array}$ & Mínimo & Mediana & Máximo \\
\hline$U_{S L}[\mathrm{~m} / \mathrm{s}]$ & 1.16 & 1.51 & 0.0006 & 0.48 & 7.25 \\
\hline$U_{S G}[\mathrm{~m} / \mathrm{s}]$ & 7.83 & 10.87 & 0.02 & 3.69 & 69.56 \\
\hline$\mu_{L}[\mathrm{mPa} . \mathrm{s}]$ & 45.0 & 145.8 & 0.74 & 4.5 & 1118.2 \\
\hline$D[\mathrm{~m}]$ & 0.05 & 0.03 & 0.02 & 0.05 & 0.15 \\
\hline$\varepsilon[\mathrm{m}]$ & $3.18 \mathrm{E}-06$ & $1.03 \mathrm{E}-05$ & 0.00 & $1.16 \mathrm{E}-06$ & $4.57 \mathrm{E}-05$ \\
\hline$d p / d z[\mathrm{~Pa} / \mathrm{m}]$ & 2628.65 & 4399.26 & 1.18 & 750.24 & 37420.96 \\
\hline
\end{tabular}

\section{PARÁMETROS ESTADÍSTICOS}

Para comparar el gradiente de presión calculado con los diferentes modelos con respecto al gradiente de presión reportado experimentalmente, se utilizan los siguientes parámetros estadísticos: $E_{1}$ es el error porcentual promedio, $E_{2}$ es el error porcentual absoluto promedio, $E_{3}$ es la desviación estándar del error porcentual promedio, los cuales son definidos como:

$$
\begin{aligned}
& E_{1}=\frac{1}{n} \sum_{i=1}^{n} r_{i} \\
& E_{2}=\frac{1}{n} \sum_{i=1}^{n}\left|r_{i}\right| \\
& E_{3}=\sqrt{\frac{1}{n-1} \sum_{i=1}^{n}\left(r_{i}-E_{1}\right)^{2}}
\end{aligned}
$$

Siendo $r_{i}=\left(\left(V_{c a l}-V_{\text {exp }}\right) / V_{\text {exp }}\right) 100$ y $n$ el número de datos experimentales.

El error porcentual promedio $E_{1}$ es una medida de la concordancia entre los valores calculados y experimentales, este indica en promedio el grado de sobrepredicción (valores positivos) 0 subpredicción (valores negativos) de los valores calculados con respecto a los experimentales. Similarmente, el error porcentual absoluto promedio $E_{2}$ es una medida de la concordancia entre los valores calculados y experimentales. Sin embargo, los errores positivos y negativos no se cancelan entre sí. Por esta razón, el error porcentual absoluto promedio es considerado un parámetro clave para evaluar la capacidad de predicción de modelos y correlaciones. La desviación estándar del error porcentual promedio $E_{3}$ indica el grado de dispersión de los errores con respeto al promedio.

\section{RESULTADOS Y DISCUSIÓN}

\section{Estudio de la densidad de mezcla}

La Fig. 1 muestra el comportamiento de las cuatros ecuaciones seleccionadas para evaluar la densidad de las mezclas bifásicas, la densidad del gas y la densidad del líquido. Los datos de densidad de mezcla se dividen en intervalos de velocidades superficiales del líquido, tal como se indica a continuación: $(0.001$ a $0.01 \mathrm{~m} / \mathrm{s}),(0.01$ a $0.1 \mathrm{~m} / \mathrm{s}),(0.1$ a $1 \mathrm{~m} / \mathrm{s}),(1$ a $10 \mathrm{~m} / \mathrm{s})$. En la leyenda se identifica cada intervalo con el valor inferior. Para mostrar las tendencias y lograr una mejor visualización, solo se graficaron un décimo de los datos experimentales, aproximadamente 250 puntos.

Se observa que las cuatro ecuaciones presentan un comportamiento adecuado, al aumentar la velocidad superficial del líquido se incrementa la densidad de la mezcla y tiende a la densidad del líquido. Ninguno de los modelos presenta valores mayores a la densidad del líquido, ni menores a la densidad del gas. Al aumentar la velocidad superficial del gas, disminuye la densidad de la mezcla y 
combinado con valores bajo de densidad de líquido la densidad de la mezcla tiende a la densidad del gas.
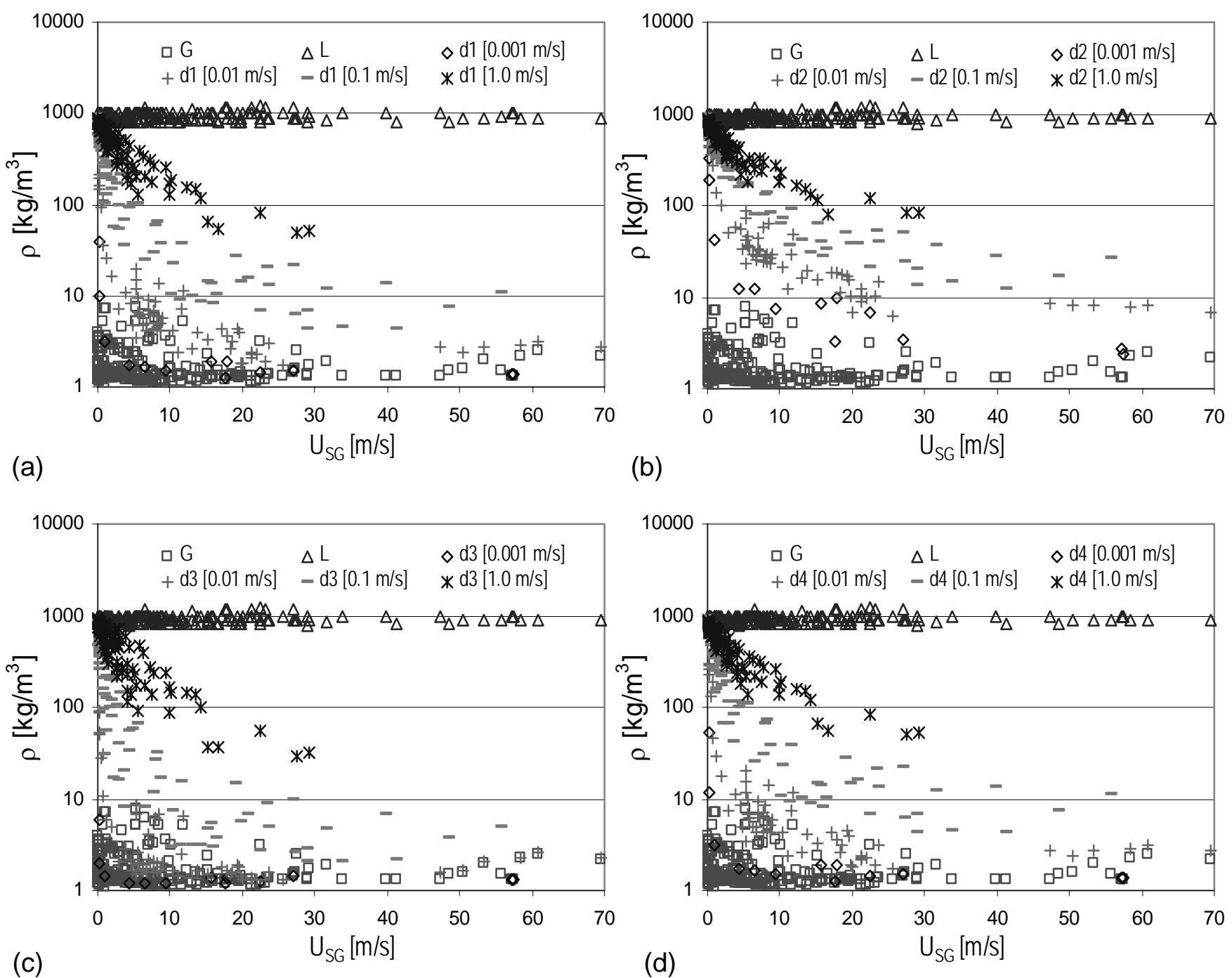

Fig. 1: Modelos Densidad de Mezcla (a) Beattie y Whalley (1982), (b) Ouyang (1998), (c) Dukler et al. (1964) y (d) Oliemans (1976)

En general, la ecuación presentada por Ouyang (1998) tiene los valores más altos de densidad de mezcla, mientras que los valores más bajos son reportados por la ecuación de Dukler et al. (1964). Las ecuaciones de Oliemans (1976) y Beattie y Whalley (1982) presentan valores intermedios entre la de Ouyang (1998) y Dukler et al. (1964). Los valores de densidad de mezcla calculados con las ecuaciones de Oliemans (1976) y Beattie y Whalley (1982) presentan entre ellas una diferencia absoluta promedio de $8.15 \%$.

En la Tabla 4, se presenta la evaluación de la precisión de los 76 modelos de gradiente de presión, al comparar los valores calculados con los valores experimentales, frente a toda la base de datos.

Al evaluar el efecto de la densidad de mezcla en la predicción del gradiente de presión de todos los modelos frente a la base de datos, se observa que los 5 mejores modelos utilizan las ecuaciones de densidad de Oliemans (1976) y de Beattie y Whalley (1982); con errores absoluto promedios que varían entre $25.7 \%$ y $28.4 \%$. Mientras que, todos los modelos que utilizan las ecuaciones de Ouyang (1998) y Dukler et al. (1964) presentan errores absolutos promedios superiores al $41 \%$. 
Tabla 4: Comparación de la precisión en la predicción del gradiente de presión de los modelos frente a los 2415 datos experimentales

\begin{tabular}{|c|c|c|c|c|c|c|c|}
\hline Modelo & $\mathrm{E} 1[\%]$ & E2 [\%] & E3 [\%] & Modelo & E1[\%] & E2 [\%] & E3 [\%] \\
\hline Dukler-d1 & -5.8 & 25.7 & 49.9 & Dukler-d3 & 90.9 & 104.5 & 251.7 \\
\hline Oliemans-d1 & -2.7 & 25.9 & 50.7 & Einstein-d2 & 82.0 & 104.7 & 282.6 \\
\hline Oliemans-d4 & -9.1 & 26.7 & 48.9 & Oliemans-d3 & 98.5 & 111.5 & 257.1 \\
\hline Fourar-d1 & 0.9 & 27.3 & 57.5 & Fourar-d3 & 108.0 & 119.8 & 281.0 \\
\hline Dukler-d4 & -11.4 & 28.4 & 49.3 & McAdams-d3 & 100.6 & 125.8 & 279.0 \\
\hline Fourar-d4 & -5.1 & 29.4 & 56.6 & Lin-d3 & 149.7 & 166.3 & 394.0 \\
\hline Beattie-d4 & 11.9 & 30.1 & 58.0 & Beattie-d3 & 158.2 & 167.1 & 350.6 \\
\hline Beattie-d1 & 21.5 & 36.1 & 66.1 & Bankoff-d3 & 177.4 & 188.2 & 409.4 \\
\hline McAdams-d1 & -3.6 & 36.7 & 67.5 & Vermeulen-d2 & 180.9 & 204.4 & 996.3 \\
\hline Bankoff-d4 & 13.7 & 37.1 & 74.8 & Cicchitti-d4 & 251.0 & 259.8 & 719.6 \\
\hline McAdams-d4 & -8.6 & 37.4 & 65.0 & Cicchitti-d1 & 274.1 & 280.3 & 743.1 \\
\hline Lin-d4 & 7.7 & 38.6 & 76.8 & Cengel-d2 & 269.2 & 290.9 & 638.8 \\
\hline Hoogendoorn-d1 & -27.3 & 40.2 & 51.6 & Soot-d4 & 468.9 & 476.4 & 1596.6 \\
\hline Beattie-d2 & -35.0 & 41.1 & 37.6 & Taylor-d4 & 481.7 & 486.2 & 1597.2 \\
\hline Lin-d1 & 15.3 & 41.5 & 86.7 & Oglesby-d4 & 482.2 & 486.3 & 1594.6 \\
\hline Bankoff-d1 & 23.3 & 41.6 & 85.6 & Hatschek-d2 & 467.7 & 486.7 & 2904.7 \\
\hline Bankoff-d2 & -40.2 & 41.8 & 30.7 & Richardson-d4 & 486.5 & 489.9 & 1596.0 \\
\hline Hoogendoorn-d4 & -30.7 & 42.2 & 50.9 & Soot-d1 & 494.9 & 500.0 & 1616.0 \\
\hline Arrhenius-d1 & -31.0 & 42.6 & 51.2 & Einstein-d4 & 504.1 & 506.7 & 1598.2 \\
\hline Lin-d2 & -39.7 & 42.9 & 36.4 & Oglesby-d1 & 509.8 & 512.3 & 1613.9 \\
\hline Arrhenius-d4 & -33.8 & 44.4 & 50.7 & Taylor-d1 & 510.0 & 512.8 & 1616.9 \\
\hline Fourar-d2 & -45.6 & 47.0 & 32.2 & Richardson-d1 & 515.1 & 517.3 & 1615.6 \\
\hline Oliemans-d2 & -46.5 & 47.6 & 32.1 & Einstein-d1 & 536.0 & 537.6 & 1618.6 \\
\hline Cicchitti-d2 & 11.5 & 48.4 & 97.8 & Vermeulen-d4 & 598.1 & 600.6 & 1835.2 \\
\hline Dukler-d2 & -47.9 & 49.1 & 31.9 & Vermeulen-d1 & 629.6 & 631.1 & 1854.6 \\
\hline McAdams-d2 & -48.5 & 50.1 & 33.9 & Cengel-d4 & 693.8 & 695.5 & 1651.0 \\
\hline Bingham-d1 & -40.8 & 52.0 & 52.7 & Cengel-d1 & 738.9 & 739.9 & 1675.8 \\
\hline Bingham-d4 & -43.2 & 53.4 & 51.8 & Cicchitti-d3 & 846.7 & 855.7 & 2183.3 \\
\hline Hoogendoorn-d2 & -60.5 & 61.2 & 28.0 & Hatschek-d4 & 958.8 & 960.1 & 3342.5 \\
\hline Arrhenius-d2 & -62.4 & 63.1 & 28.4 & Hatschek-d1 & 1012.0 & 1012.8 & 3375.9 \\
\hline Bingham-d2 & -71.3 & 71.8 & 21.1 & Soot-d3 & 1220.7 & 1229.5 & 3253.8 \\
\hline Arrhenius-d3 & 36.9 & 84.0 & 188.3 & Oglesby-d3 & 1242.8 & 1249.7 & 3257.4 \\
\hline Hoogendoorn-d3 & 45.4 & 86.0 & 193.9 & Taylor-d3 & 1250.6 & 1258.0 & 3272.0 \\
\hline Soot-d2 & 56.0 & 86.4 & 281.9 & Richardson-d3 & 1257.1 & 1263.9 & 3270.5 \\
\hline Taylor-d2 & 65.3 & 91.3 & 281.4 & Einstein-d3 & 1300.8 & 1307.5 & 3303.0 \\
\hline Bingham-d3 & 25.4 & 92.9 & 190.6 & Vermeulen-d3 & 1373.1 & 1379.5 & 3367.6 \\
\hline Oglesby-d2 & 67.7 & 93.9 & 281.4 & Cengel-d3 & 1518.9 & 1525.3 & 3279.7 \\
\hline Richardson-d2 & 69.8 & 94.6 & 281.3 & Hatschek-d3 & 1962.1 & 1970.2 & 4758.2 \\
\hline
\end{tabular}

\section{Estudio de la Viscosidad de Mezcla}

Las 19 ecuaciones de viscosidad de mezcla pueden ser divididas en tres grupos para su estudio, de acuerdo con su comportamiento respecto de la viscosidad del líquido y del gas. La ecuación de viscosidad de mezcla de Oliemans (1976), presenta un comportamiento intermedio a dos de los grupos. En la Fig. 2, se presentan casos tipos de los tres grupos más la ecuación de Oliemans (1976). En todos los casos, se grafica la viscosidad del líquido y la viscosidad de mezcla de acuerdo con la velocidad superficial del líquido en función de la velocidad superficial del gas. Los datos de viscosidad se dividen en intervalos de velocidades superficiales del líquido, tal como se indica a continuación: $(0.001$ a $0.01 \mathrm{~m} / \mathrm{s}),(0.01$ a $0.1 \mathrm{~m} / \mathrm{s}),(0.1$ a $1 \mathrm{~m} / \mathrm{s}),(1$ a $10 \mathrm{~m} / \mathrm{s})$. En la leyenda se identifica cada intervalo con el valor inferior. 
El primer grupo está constituido por las ecuaciones que conducen a resultados intermedios de viscosidades de mezcla entre la del gas y la del líquido. En este grupo se encuentran las ecuaciones de McAdams et al. (1942), Bankoff (1960), Dukler et al. (1964), Beattie y Whalley (1982), Lin et al. (1991) y Fourar y Bories (1995). Como ejemplo se graficó el comportamiento de la viscosidad reportada por Fourar y Bories (1995). En este grupo las ecuaciones son adiciones de términos que ponderan de manera distinta la viscosidad del líquido y la del gas; utilizan las fracciones volumétricas de líquido con y sin deslizamiento, la relación de la masa de gas y masa total como se muestra en la Tabla 1.
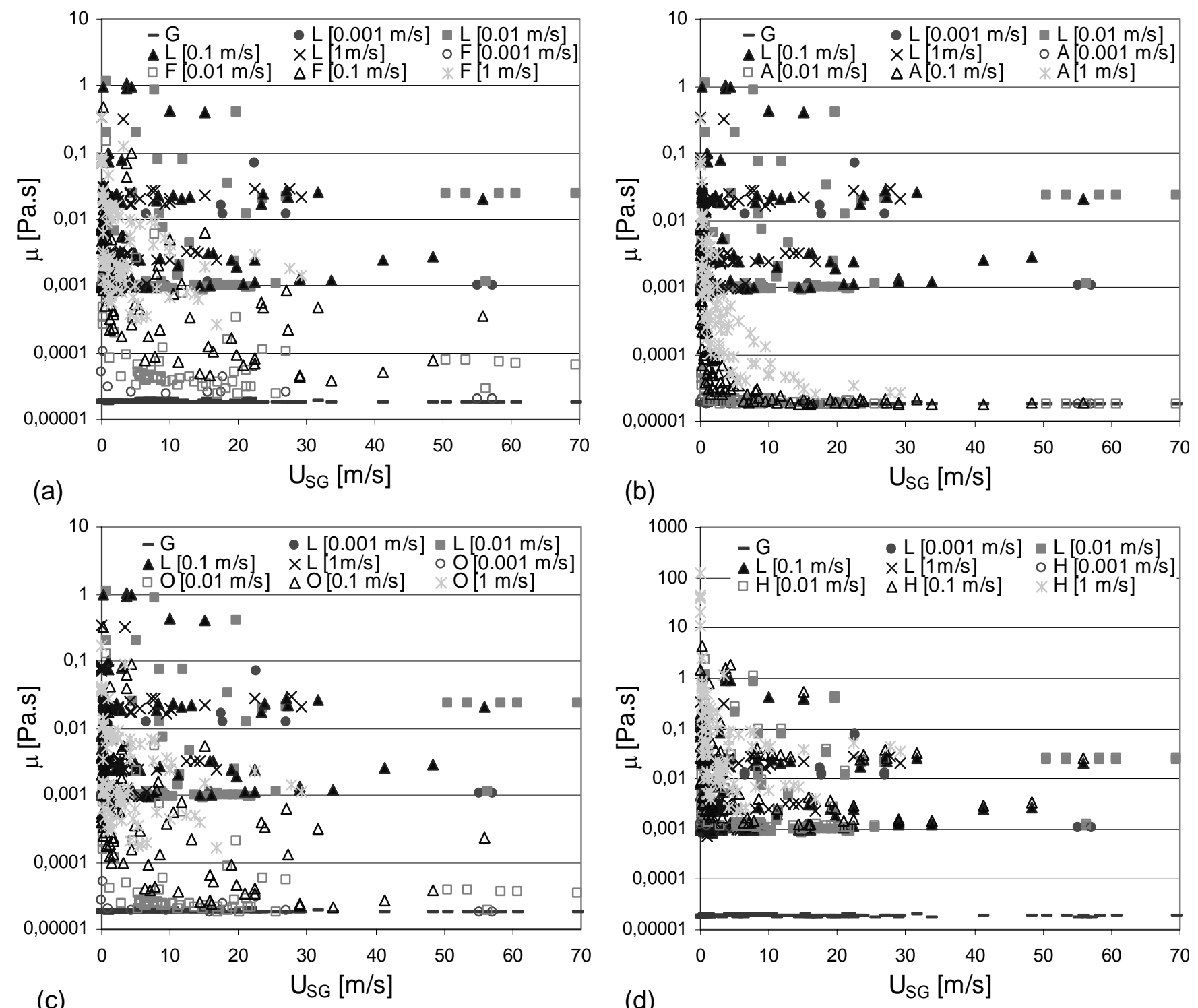

Fig. 2:. Viscosidades de Gas, de Líquido y de Mezcla: (a) Grupo 1 [Fourar y Bories, 1995], (b) Grupo 2 [Arrhenius, 1887], (c) Oliemans (1976), (d) Grupo 3 [Hatschek, 1928]

El segundo grupo está integrado por ecuaciones que tienen un comportamiento similar al del primer grupo, con valores intermedios entre las viscosidades del líquido y del gas, pero los valores se aproximan más a la viscosidad del gas a partir de los $20 \mathrm{~m} / \mathrm{s}$ de velocidad superficial del gas en comparación con los del primer grupo. Las ecuaciones que reportan este comportamiento son las de Arrhenius (1887) y Hoogendoorn (1959). Como ejemplo se muestra en la Fig. 2 el resultado de la ecuación de Arrhenius (1887); la cual utiliza la fracción de líquido sin deslizamiento, reporta valores más bajos de viscosidad de mezcla que la ecuación de Hoogendoorn (1959), que tiene la misma forma pero utiliza la fracción de líquido con deslizamiento. 
La ecuación de Oliemans (1976), reporta valores cercanos a la viscosidad del gas para velocidades superficiales del líquido entre $0.001 \mathrm{~m} / \mathrm{s}$ y $0.01 \mathrm{~m} / \mathrm{s}$, independientemente de la velocidad superficial del gas. Este mismo comportamiento se presenta para velocidades superficiales del gas mayores a $30 \mathrm{~m} / \mathrm{s}$ para el intervalo de velocidades superficiales de líquido entre $0.01 \mathrm{~m} / \mathrm{s}$ y $0.1 \mathrm{~m} / \mathrm{s}$. En este sentido, la ecuación de Oliemans (1976), puede ser considerada un caso intermedio entre los dos grupos anteriores. Esta ecuación de viscosidad de mezcla es la única que combina las fracciones volumétricas de líquido con y sin deslizamiento.

El tercer grupo está compuesto por 10 de las ecuaciones empleadas en este trabajo, la viscosidad de mezcla tiende al valor de la viscosidad del líquido o mayores a ella. En este grupo están las ecuaciones de Bingham (1906), Einstein (1909), Hatschek (1928), Taylor (1932), Richardson (1933), Vermeulen et al. (1955), Cicchitti et al. (1960), Cengel (1967), Soot (1971) y Oglesby (1979). Como un caso representativo de este grupo en la Fig. 2 se presenta la ecuación de Hatschek (1928). La mayoría de las ecuaciones de este grupo utilizan la fracción volumétrica de líquido sin deslizamiento para evaluar de distintas formas la viscosidad de la mezcla. Los resultados presentan valores muy similares a la viscosidad del líquido. La ecuación de Bingham (1906), presenta valores mayores a los de la viscosidad del líquido. La ecuación de Cicchitti et al. (1960) presenta los valores más bajos de este grupo para el intervalo de velocidades superficiales del líquido entre $0.001 \mathrm{~m} / \mathrm{s}$ y $0.01 \mathrm{~m} / \mathrm{s}$ y velocidades superficiales del gas mayores a $35 \mathrm{~m} / \mathrm{s}$. El resto de las ecuaciones presentan una diferencia absoluta promedio menor al $5 \%$ de la viscosidad de mezcla respecto de la viscosidad del líquido.

Al evaluar el error absoluto promedio de la base de datos, para la caída de presión, se observa que los mejores resultados se obtienen utilizando las ecuaciones de viscosidad de mezcla en las cuales se utilizan la fracción volumétrica de líquido sin deslizamiento, y la ecuación de Oliemans (1976) que utiliza una combinación de las fracciones volumétricas de líquido con y sin deslizamiento. Las ecuaciones de viscosidad de mezcla que solo utilizan la fracción volumétrica del líquido con deslizamiento, presentan errores mayores al 37\%. La mejor precisión la obtiene el modelo de Duklerd1 con un error absoluto promedio de $25.7 \%$, seguido del modelo de Oliemans-d1 con un error absoluto promedio de $25.9 \%$. La peor precisión la presenta el modelo de Hatschek-d3 con un error absoluto promedio cercano al $2000 \%$.

\section{CONCLUSIONES}

Al evaluar la precisión de los 76 modelos para determinar el gradiente de presión frente a la base de datos, se puede concluir que los mejores resultados se obtienen con modelos que utilizan las ecuaciones de densidad de Oliemans (1976) y Beattie y Whalley (1982). Al realizar el estudio por viscosidad de mezcla para la caída de presión, en general se observa que los mejores resultados se obtienen utilizando ecuaciones de viscosidad de mezcla que utilizan la fracción volumétrica de líquido sin deslizamiento. Aquellas ecuaciones de viscosidad de mezcla que solo utilizan la fracción volumétrica de líquido con deslizamiento presentan los mayores errores absolutos promedios. De los resultados obtenidos se evidencia, que el modelo de gradiente de presión que considera al flujo bifásico como una mezcla homogénea, es altamente sensible a la definición de la ecuación de viscosidad de mezcla, siendo el modelo de Dukler-d1 el más preciso.

\section{AGRADECIMIENTOS}

Janneth García, agradece al DID-USB por el apoyo económico otorgado a través del proyecto S1-INCAI-018-06. Francisco García, agradece al CDCH-UCV por el apoyo económico otorgado a través de los proyectos de investigación 08.00.6245.2006 y 08.00.5653.2007 y la ayuda institucional 08.00.6681.2007.

\section{NOMENCLATURA}

$A:$ área $\left(\mathrm{m}^{2}\right)$
$D:$ diámetro $(\mathrm{m})$
$E:$ parámetros estadísticos $(-)$




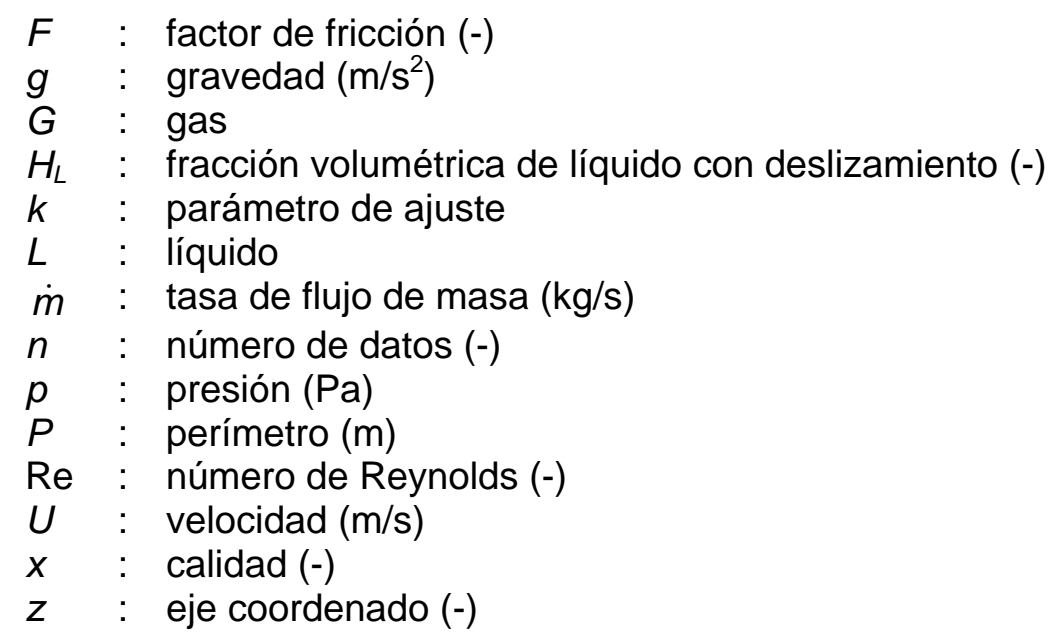

\section{Símbolos Griegos}

$\beta \quad$ : factor de corrección (-)

$\lambda_{L} \quad$ : fracción volumétrica de líquido sin deslizamiento $(-)$

$\mu \quad$ : viscosidad dinámica (Pa s)

$\theta \quad$ : ángulo de inclinación $\left({ }^{\circ}\right)$

$\rho:$ densidad $\left(\mathrm{kg} / \mathrm{m}^{3}\right)$

$\tau \quad:$ esfuerzo cortante (Pa m)

\section{Subíndices}

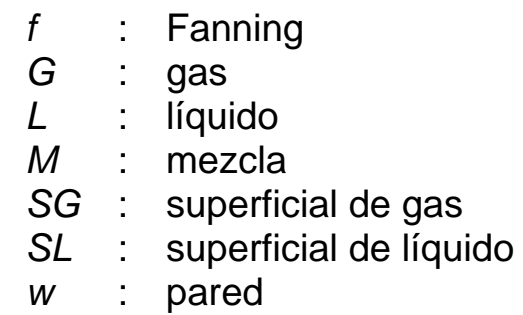

\section{REFERENCIAS}

Arrhenius, S.; On the Internal Friction of Solutions in Water, Zeitschrift für Physikalische Chemie (Leipzig): 1, 285-298 (1887).

Bankoff, S.; A Variable-Density, Single-Fluid Model for Two-Phase Flow with Particular Reference To Steam-Water Flow, Journal of Heat Transfer: 82, 265-272 (1960).

Beattie, D. y P. D. Whalley; A Simple Two-Phase Frictional Pressure Drop Calculation Method, Int. J. of Multiphase Flow: 8(1), 83-87 (1982).

Bingham, E.; Viscosity and Fluidity, American Chemical Journal: 35, 95-217 (1906).

Cengel, J.; Viscosity of Liquid-Liquid Dispersions in Laminar and Turbulent Flow, Ph. D, Thesis, Oregon State Univ. Abst. No. 67-11805 (1967).

Cicchitti, A.; y otros cuatro autores; Two-Phase Cooling Experiments-Pressure Drop, Heat Transfer and Burnout Measurements, Energi Nucl: 7(6), 407-425 (1960).

Dukler, A., M. Wicks III y R.Cleveland; Frictional Pressure Drop in Two-Phase Flow: B. An Approach through Similarity Analysis, AIChE Journal: 10(1), 44-51 (1964). 
Eaton, B. y otros cuatro autores; The Prediction of Flow Patterns, Liquid Holdup and Pressure Losses Occurring During Continuous Two-Phase Flow in Horizontal Pipelines, Trans. AIME: 815 (1967).

Eaton, B.; The Prediction of Flow Patterns, Liquid holdup and pressure losses occurring during continuous two-phase flow in horizontal pipelines. Ph.D. Thesis, University of Texas (1966).

Einstein, A.; A New Definition of Molecular Dimensions. Annalen der Physik: 4, 289-306 (1909).

Fourar, M. y S. Bories; Experimental Study of Air-Water Two-Phase Flow through a Fracture (Narrow Channel), Int. J. of Multiphase Flow: 21(4), 621-637 (1995).

García, F. y otros cinco autores; Power Law and Composite Power Law Friction Factor Correlations for Laminar and Turbulent gas-Liquid Flow in Horizontal Pipelines, Int. J. of Multiphase Flow: 29(10), 1605-1624 (2003).

García, F. y otros tres autores; Friction factor improved correlations for laminar and turbulent gasliquid flow in horizontal pipelines, Int. J. of Multiphase Flow: 33(12), 1320-1336 (2007).

Haoulo, M., J. Soto y F. García; Gradiente de presión de flujo de gas y líquido en tuberías horizontales considerando el efecto de las propiedades de mezcla, Revista de la Facultad de Ingeniería de la UCV: 20(4), 5-22 (2005).

Hatschek, E.; The Viscosity of Liquids. London: Bell \& Sons Ltd (1928).

Hoogendoorn, C.; Gas-Liquid Flow in Horizontal Pipes, Chem. Eng. Sci.: 9, 205-217 (1959).

Lin, S. y otros cuatro autores; Local frictional pressure drop during vaporization for R-12 through capillary tubes, Int. J. of Multiphase Flow: 17(1), 95-102 (1991).

McAdams, W., W. Woods y L. Heroman; Vaporization Inside Horizontal Tubes, Trans. ASME: 64, 193-200 (1942).

Moreno, J. y, J. Thome; Flow pattern based two-phase frictional pressure drop model for horizontal tubes. Part I: Diabatic and adiabatic experimental study, Int. J. of Heat and Fluid Flow: 28(5), 10491059 (2007a).

Moreno, J. y, J. Thome; Flow pattern based two-phase frictional pressure drop model for horizontal tubes, Part II: New phenomenological model, Int. J. of Heat and Fluid Flow: 28(5), 1060-1072 (2007b).

Oglesby, K.; An Experimental Study on the Effects of Oil Viscosity Mixture Velocity and Water Fraction on Horizontal Oil-Water Flow, TEGM The University of Tulsa (1979).

Oliemans, R.; Two Phase Flow in gas-Transmission Pipelines, ASME paper 76-Pet-25, presented at Pet. Div. ASME meeting, Mexico (1976).

Ouyang, L.; Single Phase and Multiphase Fluid Flow in Horizontal Wells, PhD Dissertation Thesis, Department of Petroleum Engineering, School of Earth Sciences, Stanford University (1998).

Pereyra, E. y otros tres autores; Validación del modelo mecanicista de gradiente de presión para flujo tapón en un sistema crudo/gas, INT-8894,2001. PDVSA INTEVEP (2001).

Richardson, E.; On the Viscosity of Emulsions, Kolloid Zeitschrift: 65, 32-37 (1933).

Saisorn, S. y S. Wongwises; An inspection of viscosity model for homogeneous two-phase flow pressure drop prediction in a horizontal circular micro-channel, Int. Communications in Heat and Mass Transfer: 35(7), 833-838 (2008). 
Soot, P.; A Study of Tow-Phase Liquid-Liquid Flow in Pipes, Ph. D. Thesis, Oregon Statte University, Abst. No. 71-2479 (1971).

Spedding, P., E. Benard y G. Donnelly; Prediction of pressure drop in multiphase horizontal pipe flow, Int. Communications in Heat and Mass Transfer: 33(9), 1053-1062 (2006).

Taylor, G.; The Viscosity of Fluid Containing Small Drops of Another Fluid, Proceeding of the Royal Society of London: 138A(834), 41-48 (1932).

Vermeulen, T., G. M. Williams y G. E., Langlois; Interfacial Area in Liquid-Liquid and Gas-Liquid Agitation, Chm. Eng. Progress: 51, 85F-94F (1955).

Wallis, G.; One Dimensional Two-Phase Flow, 18-26, McGraw-Hill, New York (1969). 